\title{
TRIPLE GALAXIES AND A HIDDEN MASS PROBLEM
}

\author{
I.D.Karachentsev, V.E.Karachentseva, V.S.Lebedev \\ Special Astrophysical Observatory, USSR Academy of Sciences \\ st. Zelenchukskaya, Stavropol Territory, 357147, USSR
}

\begin{abstract}
We consider a homogeneous sample of 84 triple systems of galaxies with components brighter than $m=15.7$, located in the northern sky and satisfying an isolation criterion with respect to neighboring galaxies in projection. The distributions of basic dynamical parameters for triplets have median values as follows: radial velocity dispersion $133 \mathrm{~km} / \mathrm{s}$, mean harmonic radius $63 \mathrm{kpc}$, absolute magnitude of galaxies $\mathrm{M}_{B}=-20.38$, crossing time $\tau=0.04 \mathrm{H}^{-1}$. For different ways of estimation the median mass-to-luminosity ratio is (20 $-30) f_{\odot}$.

A comparison of the last value with the ones for single and binary galaxies shows the presence of a virial mass excess for triplets by a factor 4 . The mass-to-luminosity ratio is practically uncorrelated with linear size of triplets or with morphological types of their components.

We note that a significant part of the virial excess may be explained by the presence of nonisolated triple configurations in the sample, which are produced by debris of more populous groups of galaxies.
\end{abstract}

\section{INTRODUCTION}

On comparing a recent survey on dark matter in the Universe (Trimble, 1987) with the preceding one covering the topic (Faber, Gallagher, 1979), the following tendencies are apparent.

The idea, that at rather large scales $(L>50 \mathrm{kpc})$ hidden mass in the Universe dominates over visible matter, is becoming more and more popular. Inflationary cosmological theories have played a significant part here. Concerning observational arguments, their quantity and quality are higher, as the scale or population of the system of galaxies considreed is larger. But information on the nature and distribution of the hidden mass is still rather primitive. The least reliable evidence seems to be that for galaxian systems of low multiplicity. According to Karachentsev (1987), the overall observational data on the most numerous $(n \approx 600)$ and homogeneous binary-galaxy sample can be explained without requiring hidden mass there, on scales $20-1200 \mathrm{kpc}$.

Therefore the need to investigate low-multiplicity systems of galaxies, including triple systems, is clear. The first, and as far as we are concerned, still unique attempt to study triple galaxies systematically was made by Karachentseva et al. (1979). A criterion of local isolation was applied to the observed distribution of galaxies brighter than $m=15.7$. 
Three galaxies were considered as forming an isolated system if 'significant' neighbors were standing at least three times as far from them, as the triplet galaxies were from one another. Significant neighbors were taken as surrounding galaxies with angular diameters not more than a factor two different from the triplet members' diameters. Such a condition selects systems with $10 \times$ and higher excess of galaxy surface density over the average background.

If we compare the criterion for isolated triplets with an analogous double-system criterion (Karachentsev, 1972), it is actually less restrictive for triple galaxies (the avoidance zone around a pair exceeded 5 component separations). Nevertheless, among 27,841 galaxies in the northern sky brighter than $m=15.7$ only 84 triplets satisfy this isolation criterion, which is 7 times smaller than the number of isolated pairs.

It is important to note that galaxy membership in a triple system was determined without recourse to radial velocity. Therefore a system might be isolated only in projection on the sky, but not three-dimensional space.

The program of radial velocity measurements for triple galaxies was performed mainly at the 6-m telescope. The results so obtained together with data from other sources were compiled in a previous article (Karachentseva et al. 1988). There a complete radial velocity summary for triple galaxies, their angular diameters and apparent magnitudes reduced to the standard isophotal diameter $\left(\mathrm{R}_{25}\right)$ and Holmberg magnitude $\left(\mathrm{m}_{H o}^{c}\right)$ system, and also more detailed determinations of morphological types of the galaxies were presented. We will consider below how triple systems are distributed according to linear dimensions, velocity dispersion, total luminosity and other characteristics bearing on the dynamical conditions of triple galaxies.

\section{MAIN PARAMETERS}

In describing triple galaxies we will use two mutually complementary approaches. a) Triplet as a unit dynamical system. It is characterized by the following values: centroid mean radial velocity

$$
<\mathrm{v}>=\sum_{k=1}^{3} \mathrm{v}_{k} / 3
$$

where $v_{k}$ is the component radial velocity, corrected for the solar motion using the standard formula (de Vaucouleurs et al. 1976);

rms velocity of galaxies relative to the centre,

$$
S_{v}=\left[\sum_{k=1}^{3}(V-<V>)^{2}\right]^{1 / 2}
$$

projected linear separation between components,

$$
r_{i k}=<\mathrm{V}>\mathrm{H}^{-1} \mathrm{X}_{i k}
$$

where $\mathrm{X}_{i k}$ is the angular separation, and $\mathrm{H}$ the Hubble constant, taken as $75 \mathrm{~km} / \mathrm{s} \mathrm{Mpc}$; 
average projected harmonic separation,

$$
r_{H}=\left[\sum_{i, k} r_{i k}^{-1}\right]^{-1}
$$

galaxy luminosity in solar units,

$$
L_{k}=\operatorname{dex}\left[0.4\left(5.40-M_{k}\right)\right] \text {, }
$$

where the absolute magnitude $\mathrm{M}_{k}$ is calculated using the average radial velocity $<\mathrm{V}>$, and apparent magnitude $m_{H_{0}}$ in the Holmberg system with corrections for obscuration in our Galaxy, cosmological K-effect, and internal absorption in the considered galaxy;

virial mass to total luminosity-ratio for a triplet,

$$
\mathrm{f}=\frac{3 \pi n(n-1) S^{2}{ }_{\mathrm{v}} r_{H}}{\mathrm{G} \sum_{k} L_{k}}
$$

where $\mathrm{G}$ is the gravitational constant, $\mathrm{n}=3$, and $3 \pi$ is the mean projection factor according to Limber and Mathews (1960);

a statistically unbiased estimator of the virial mass-to-luminosity ratio,

$$
f^{c}=f \frac{1-2 \sigma^{2} v}{3 S^{2} v}
$$

where $\sigma_{v}$ is the rms error of radial velocity measurement for triple galaxies;

dimensionless "crossing time" of a system,

$$
\tau=2 \mathrm{H} r_{H} / S_{\mathrm{v}}
$$

expressed in units of the Hubble time, $\mathrm{H}^{-1}$.

Integral characteristics of each triple system are presented in the summary (Karachentsev et al. 1989). The reduced data from the reference list (Karachentseva et al. 1988), were used for their calculations.

Similar calculations were repeated for triple systems with each galaxy given a weight proportional to its luminosity. In most cases differences between weighted and unweighted parameters turned out negligible.

b) Triple system as a set of pairs. Previous estimates of triple-system masses were based on the supposition that the ratio of system kinetic to potential energy $k=2 T /|U|$, does not change with time and equals unity. Simulating triple-system evolution, Anosova and Orlov (1985) showed that the virial coefficient $\mathbf{k}$ undergoes considerable fluctuations $<\Delta \mathrm{k}^{2} / \mathrm{k}^{2}>\approx 1$. Additional uncertainty in virial mass estimation would be introduced by projection factors. As Bahcall and Tremaine noted (1981), the estimation of $f$ from expression (6) is not statistically robust. Thus they suggested that $f$ be determined not according to the average harmonic radius $\left\langle r_{i k}{ }^{-1}\right\rangle$, but according to an average value $<\left(\mathrm{V}_{i}-\mathrm{V}_{k}\right) r_{i k}{ }^{2}>$. This approach corresponds to interpretation of a triple system as an ensemble of galaxy-satellite systems, revolving around the main (most massive) component. 
Formally dividing a triple system into three pairs of galaxies and for definiteness positing circular motions in the pairs, we obtain an estimate of orbital mass-to-luminosity ratio,

$$
\mathrm{f}_{i k}=\frac{32 r_{i k}\left(\mathrm{~V}_{i}-\mathrm{V}_{k}\right)^{2}}{3 \pi \mathrm{G}\left(L_{i}+L_{k}\right)}
$$

and also the unbiased estimate with correction for radial velocity measurement errors:

$$
\mathrm{f}^{c}{ }_{i k}=\mathrm{f}_{i k}\left[1-\left({\sigma_{i}}^{2}+{\sigma_{k}}^{2}\right) /\left(\mathrm{V}_{i}-\mathrm{V}_{k}\right)^{2}\right]
$$

Here the projected linear separation between galaxies is expressed through the pair's average radial velocity,

$$
r_{i k}=X_{i k} \mathrm{H}^{-1}\left(\mathrm{~V}_{i}+\mathrm{V}_{k}\right) / 2
$$

and the galaxy luminosity through its individual radial velocity.

As mentioned above, the isolation criterion for a triple system of galaxies was chosen softer than the pair criterion. According to the data of Karachentsev (1987) only $57 \%$ of binary systems are true isolated pairs, so we may expect that in the list of triple galaxies a considerable number are false ones. To verify this suggestion we used numerical simulations of the apparent galaxian distribution (Karachentsev, Shcherbanovskij, 1979). The main results of the simulation concerning the triple systems have already been discussed by Karachentseva and Karachentsev (1982).

In 127 fields, imitating the POSS prints, among 9433 galaxies brighter than $m=15.7$ 71 isolated triple systems were selected, applying the above-mentioned criterion. We used them as a reference sample when analyzing properties of catalogued triplets. Without going into details, we shall note only two important results of the simulation: A) The isolation criterion removes about $2 / 3$ of triple systems, all of whose components are brighter than the sample limit of $m=15.7$; the criterion is strongly selective, favoring "survival" of the tightest systems. B) The isolation condition is satisfied by a large number of false triple systems. Among 71 simulated triplets only 5 (or $7 \%$ ) are true physical triple systems; 31 triplets (44\%) are formed by galaxies which are members of a group or a cluster, and the remaining part of the sample (49\%) constitute optical triplets. Among the last category are 5 completely spurious triplets and 30 "semi-optical" triplets - i.e. a pair or two system members plus a background galaxy.

Let us consider the distribution of triple galaxies according to their relative radial velocities. The data are presented in Figure 1. The left histogram shows the number of catalogued triplets versus radial velocity difference for the components. The distribution of relative radial velocities for 585 isolated pairs (Karachentsev, 1972) is dashed for comparison. The right side gives analogous data for the simulated triple systems. True physical triplets are marked with double hatching, non-isolated triple galaxies, consisting of group or cluster members with single hatching, and optical systems are not hatched. 


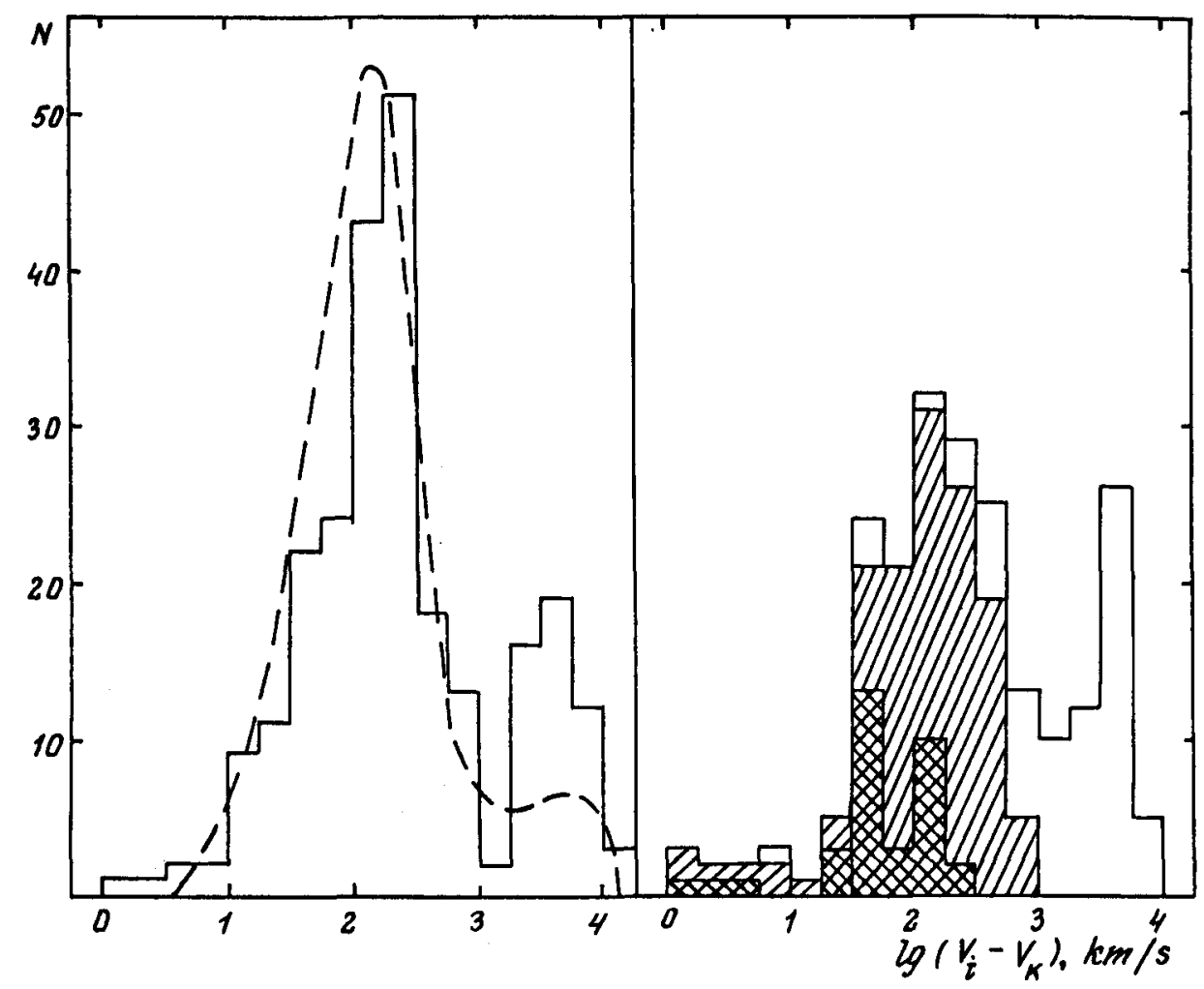

Fig. 1. Triple galaxy distribution of radial velocity difference. Catalogue triplets are to the left, simulated ones to the right. Double-hatched regions are physical systems, single-hatched are non-isolated members of groups and clusters. The dashed curve reflects the distribution for 585 isolated pairs.

As we can see, the distribution has an asymmetric shape. Besides the main maximum at $100 \mathrm{~km} / \mathrm{s}$ a secondary rise in the region $\left(10^{3}-10^{4}\right) \mathrm{km} / \mathrm{s}$ is observed. An obvious reason for the secondary peak is the contribution of optical triple systems. Pairs of galaxies from the catalogue of Karachentsev (1972), selected according to a stricter isolation criterion, also show an excess of cases in this region.

Turning to the right side of Figure 1, we may claim satisfactory correspondence between the distributions of simulated and catalogued triplets. Though optical and physical triplets overlap slightly, there is no difficulty in distinguishing between them. According to the results of numerical simulations, optical triplets dominate in the region $S_{v}>300$ $\mathrm{km} / \mathrm{s}$ or $\left(V_{i}-V_{k}\right)>600 \mathrm{~km} / \mathrm{s}$. It is different with triple systems whose members belong to some group or cluster. They overlap so completely with true isolated triplets that we don't see any simple method to separate them.

In distributions of a random variable with a large asymmetry coefficient, the sample mean depends strongly on one or two maximum values at the tail of a sample. Because of this, many authors prefer to use a distribution median instead of the average, as a more robust estimator. We also follow this approach. The distributions of radial-velocity difference for members of catalogued and simulated triplets are characterized by medians $198 \mathrm{~km} / \mathrm{s}$ and $217 \mathrm{~km} / \mathrm{s}$, respectively. For comparison we note that for 585 isolated pairs the median is considerably smaller, $130 \mathrm{~km} / \mathrm{s}$.

In Figure 2 the distributions of catalogued (left) and simulated triplets are presented according to projected linear separation $r_{i k}$. Various types of the simulated triplets are 
distinguished as in Figure 1. The dashed curve shows a smoothed distribution for 585 isolated pairs. Median values are $77 \mathrm{Kpc}$ for the catalogued triplets and $90 \mathrm{Kpc}$ for the simulated ones. Isolated pairs (dashes) are tighter systems on average; their median separation is only $30 \mathrm{Kpc}$.

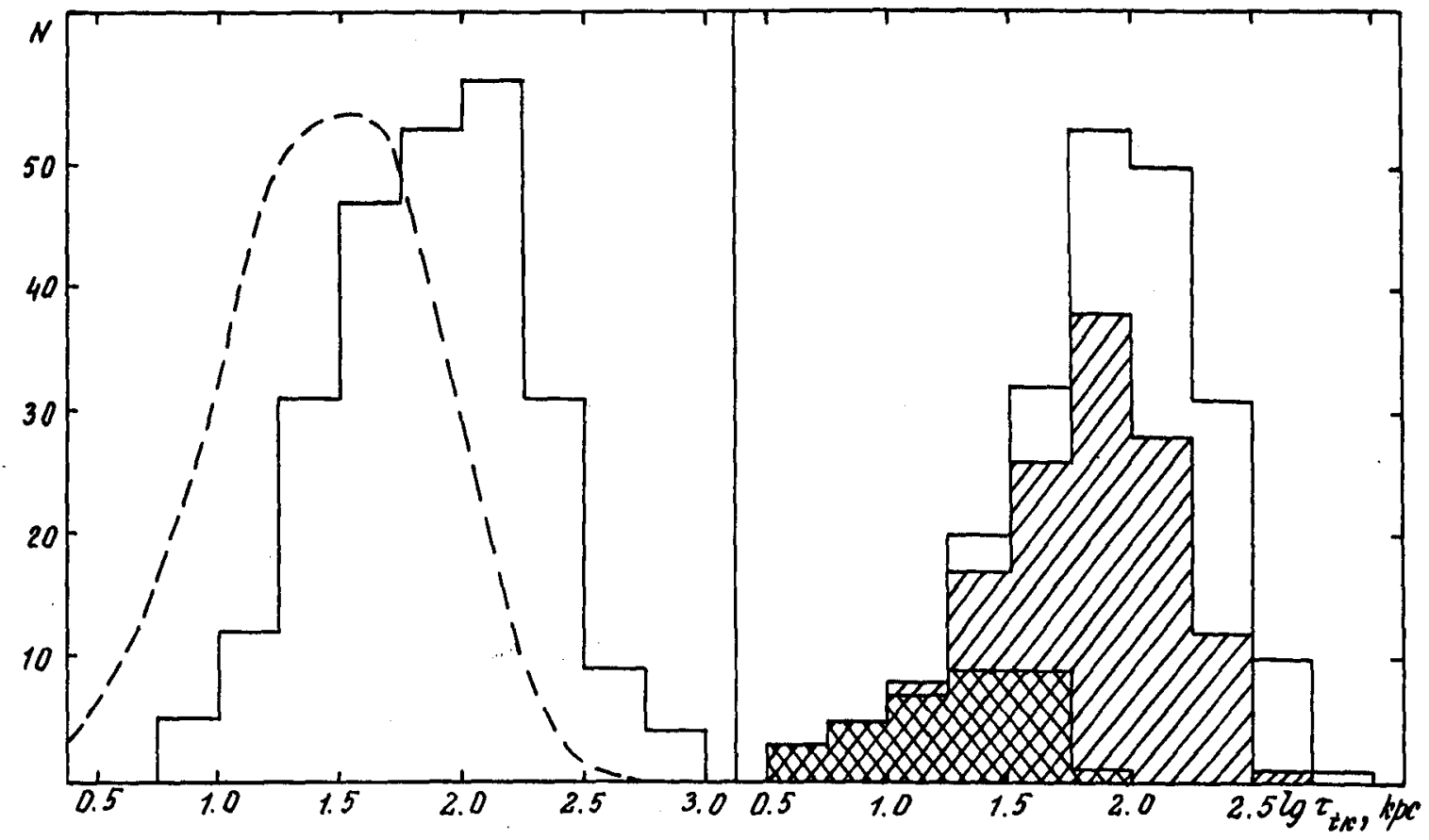

Fig. 2. Triple-system distribution in projected linear separation between the components. Hatching shows the same categories of objects as in Figure 1.

The distribution of catalogued triplets in $\mathrm{S}_{\mathrm{v}}, \mathrm{r}_{\mathrm{H}}$ - coordinates is shown in Figure 3. Triple systems in this diagram are concentrated in distinct groups, which may indicate dynamical or structural inhomogeneity of the sample. On the whole a tendency is evident for velocity dispersion to decrease for triple systems on passing from tight systems to wide ones. Such a feature is characteristic also of the $\left(V_{i}-V_{k}\right), r_{i k}$ distribution. The presence of a weak negative correlation between mutual velocities and separations of galaxies should be expected, if their motions in triple systems are close to Keplerian.

As the results of numerical simulations show, selection effects in triple systems are quite strong and various. Practically all the catalogued parameters of triple galaxies are distorted both by selection conditions and the presence of false triplets. Several examples of these will be given below. 


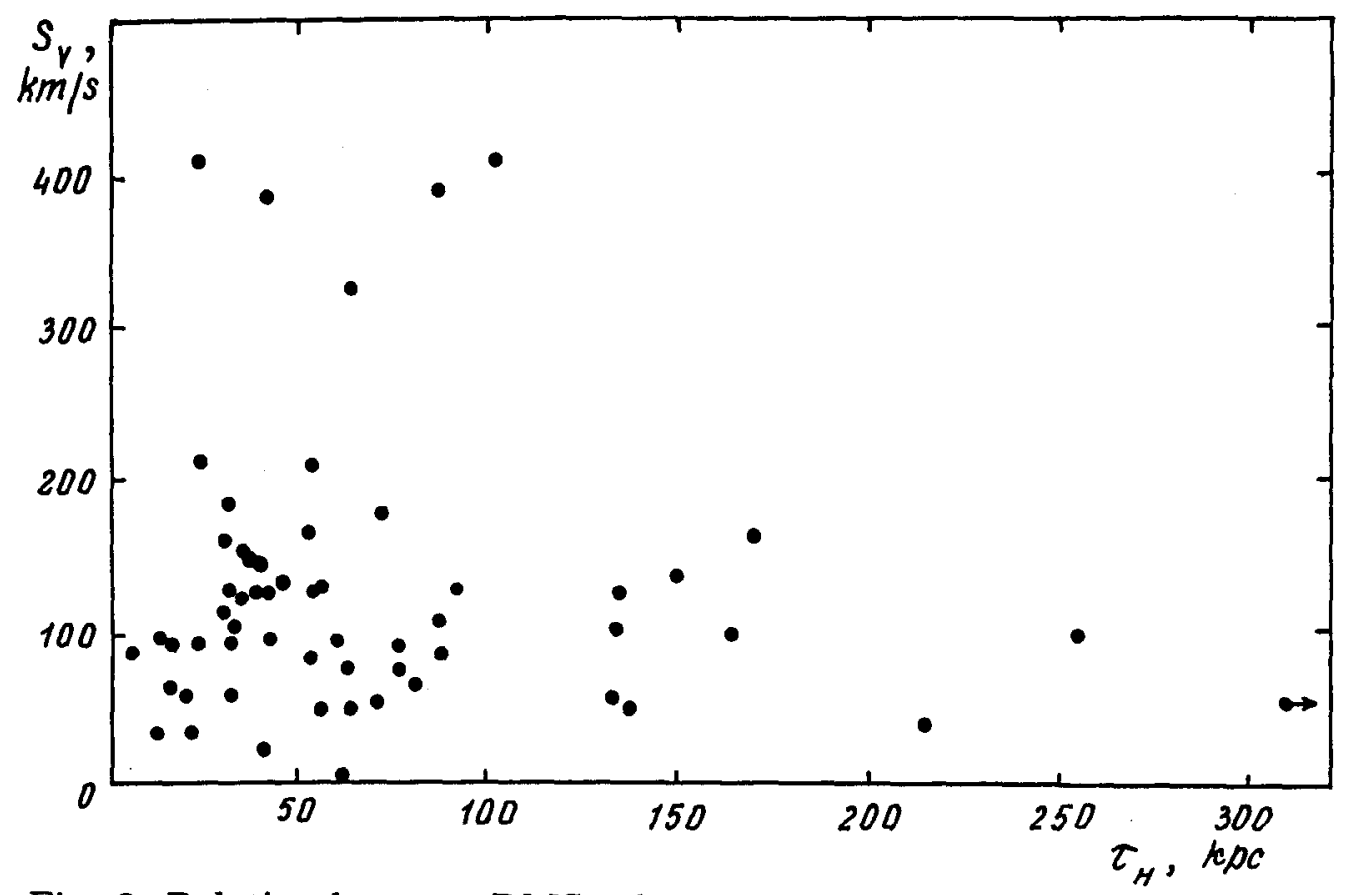

Fig. 3. Relation between RMS velocity and linear dimension for triplets.

\section{VIRIAL MASS-TO-LUMINOSITY RATIOS}

Mass-to-luminosity ratios in triplets were determined in several ways. The results are presented in Figure 4. The upper histogram (A) shows the distribution of the number of systems according to $\log f$ obtained from eq. (6). The middle histogram (W) reflects the distribution of $f$ weighting each galaxy proportionally to its luminosity. The lower diagram (P) shows the distribution of $f_{i k}$, i.e. orbital mass-to-luminosity ratio for each pair of triplet members according to eq. (9). The dashed curve shows an analogous dependence for 585 catalogued pairs. In all three histograms common features may be noted : a broad interval of the argument covering 6-7 orders of magnitude; the location of a primary maximum near $f \approx 30 f_{\odot}$, and the existence of a secondary rise in the region $\left(10^{3}-10^{5}\right) \mathrm{f}_{\odot}$.

Using the data on mutual velocities, separations and luminosities for components of the simulated triplets, we determined virial ratios $f, f_{w}$, and $f_{i k}$ for them, using the same formulae as for real systems. Though such an approach is particularly formal, it has practical sense, as it allows us to estimate the role of projection factors and the presence of background objects. The distribution medians for physical triplets $(\mathrm{T})$, group and cluster members $(G)$ and optical systems $(O)$ are shown with arrows in each of the diagrams $A$, $\mathrm{W}, \mathrm{P}$ of Figure 4. According to the results of numerical experiments we may assert, that using all the methods of f-estimation the region of a secondary maximum, $\left(10^{3}-10^{5}\right)$ $f_{\odot}$, appears to be occupied mainly by optical triplets. Spatially isolated triplets and false triple systems (of members of one group or cluster) have similar medians and are difficult to distinguish inside the common main maximum. In the $\mathrm{W}$ - and $\mathrm{P}$-histograms one could suspect such a distinction, but it is washed out by random projection factors.

Table 1 presents median estimates of the virial mass-to-luminosity ratio, obtained by different methods. Together with biased f-values we give also median $\mathrm{f}^{c}$-values, reduced for radial velocity measurement errors according to eqs. (7) and (10). To account for the 
effects of optical triple systems, we give also data for truncated samples. In one case 25 triplets with $f>10^{3} f_{\odot}$ were excluded, in the other - 30 systems, where the radial velocity difference exceeds $500 \mathrm{~km} / \mathrm{s}$ for at least one pair of components. The last line of the table shows medians for 585 pairs; the conditions $f<10^{3} f_{\odot}$ and $\left(V_{i}-V_{k}\right)<500 \mathrm{~km} / \mathrm{s}$ preserve 531 and 497 binary systems in this sample, respectively. Analysis of the data allows us to draw the following conclusions.

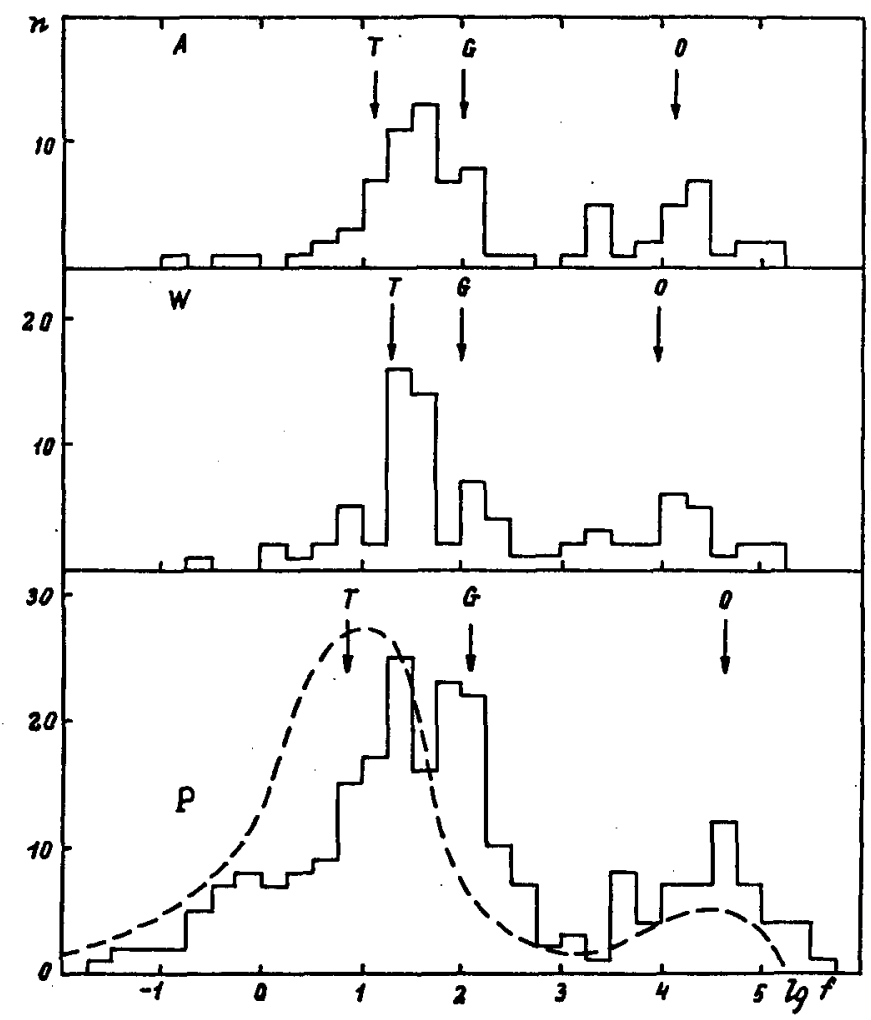

Fig. 4. Distribution of the triplets in virial mass-to-luminosity ratio in solar units. At the top, mean arithmetic estimates (A), in the middle, average weighted over luminosity $(\mathrm{W})$, and at the bottom, pair orbital mass estimations. Arrows show median values for true triplets (T), group members (G) and optical systems (O) according to the results of numerical simulation.

a) Accounting or not-accounting for radial velocity measurement errors weakly influences median estimate of virial mass-to-luminosity ratio. A typical difference detween them is only 0.07 dex.

b) The median weakly depends on how we estimate mass-to-luminosity ratio. There is an average difference of 0.04 dex between a simple arithmetic estimate (A) and the value weighted according to luminosity (W), i.e. equipartition is practically absent for giant and dwarf galaxies. Description as a sum of pairs with circular motions of galaxies gives the median close to other methods. We note that for the best agreement of all the median values in different lines of Table 1 it is sufficient to suggest elliptical orbital motions with the eccentrity, $e=0.40-0.55$.

c) From the last line one can see that triplets have a systematic excess of virial mass-to-luminosity ratio in comparison with isolated pairs. As for the whole sample of 
triple systems, so for the truncated samples the value of such excess is nearly the same and constitutes a factor of $\approx 5$. This feature is clearly seen in the lower part of Figure 4 .

d) For different ways of excluding optical systems (the condition $\mathrm{f}<10^{3} \mathrm{f}_{\odot}$ or $\Delta \mathrm{V}_{i k}<$ $500 \mathrm{~km} / \mathrm{s}$ ) and various ways to estimate virial mass $(A, W, P)$ medians $\left[f^{c}\right]_{0.5}$ are concentrated in a narrow interval [ $20-29$ ] $f_{\odot}$. Comparing them to the median of individual mass-to-luminosity ratios $\left[f_{i n d}\right]_{0.5}=5.9 f_{\odot}$, determined from 227 galaxies ( Karachentsev, 1985), we obtain the value of virial mass excess for triplets, (3.4-4.9), i.e. similar to the previous one.

e) In the initial list all triple systems were divided into types : $T$ - triangle, more or less close to an equilateral one, $\mathrm{L}$ - components are linar, $\mathrm{D}$ - two close galaxies with a distant satellite. The latter configuration is often called hierarchical. It is the most stable during dynamical evolution (Anosova, Orlov, 1985). Having arranged triplets according to their virial mass-to-luminosity ratio we didn't find any significant differences between hierarchical configurations and the rest.

f) One of the main tests for the presence of hidden mass in galaxian systems is the character of the "virial mass - system size" relation. Such data for triplets are given in Figure 5 and 6 . The first reflects the distribution of triplets according to unbiased virial mass-to-luminosity ratio and average harmonic separation. The expected increase (in the presence of hidden mass) of $\mathrm{f}^{c}\left(\mathrm{r}_{H}\right)$ is shown very weakly and has low statistical reliability.

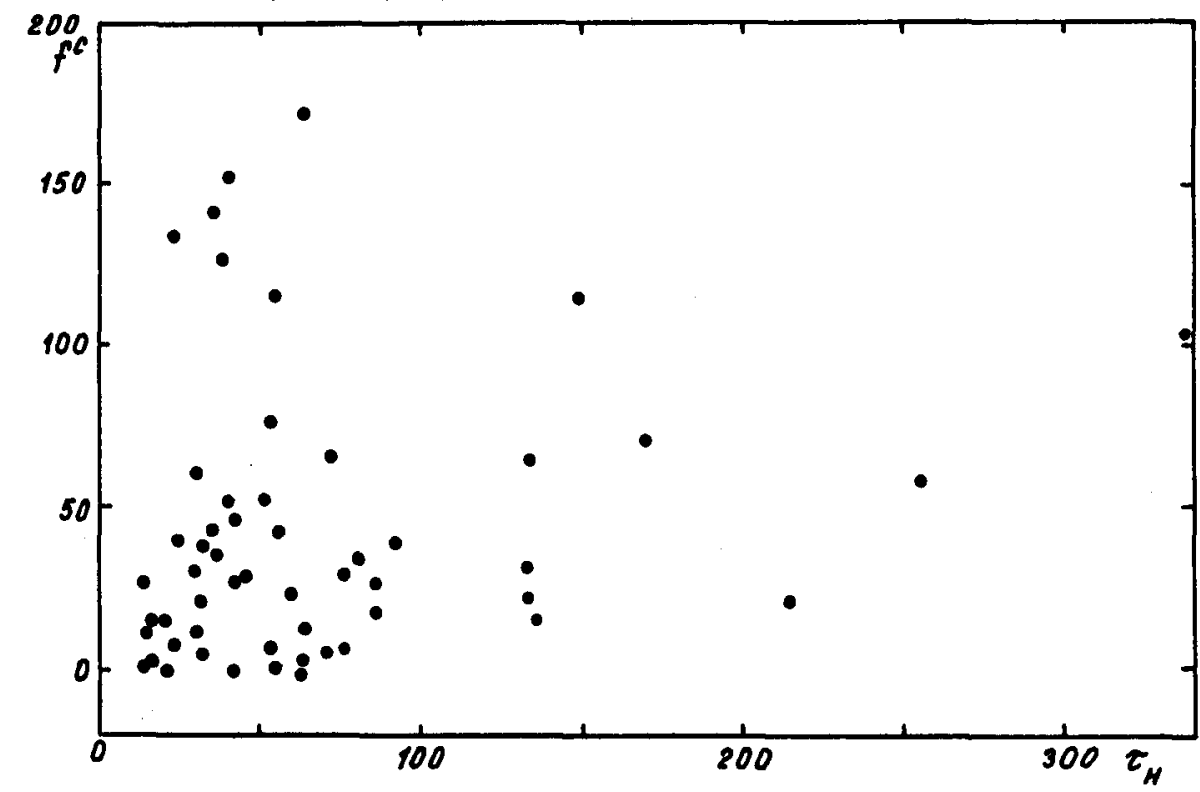

Fig. 5. Triple-system distribution according to unbiased mass-to-luminosity ratio and linear harmonic separation $(\mathrm{kpc})$.

In Figure 6 the orbital mass-to-luminosity ratio is presented versus projected linear separation for triple system components. Negative $\left.f^{c}\right]_{i k}$ values correspond to cases when velocity measurement errors exceed the radial velocity difference. Judging by the relative number of such cases and the amplitudes of negative values, velocity measurement errors do not vastly influence dynamical parameters of the sample in general. As in the previous Figure, the increase of orbital mass-to-luminosity ratio with the growth of distance between galaxies reveals only a weak trend. Among wide pairs with $r_{i k}>100 \mathrm{kpc}$ two subsystems 
can be seen : for the first the $f^{c}{ }_{i k}$ values are located in a horizontal band $[+25,-10] f_{\odot}$, for the second - at the rising branch. This subdivision of objects into two subsystems is not accompanied by any significant distinction of galaxies according to their morphological types, luminosities or other integral parameters.

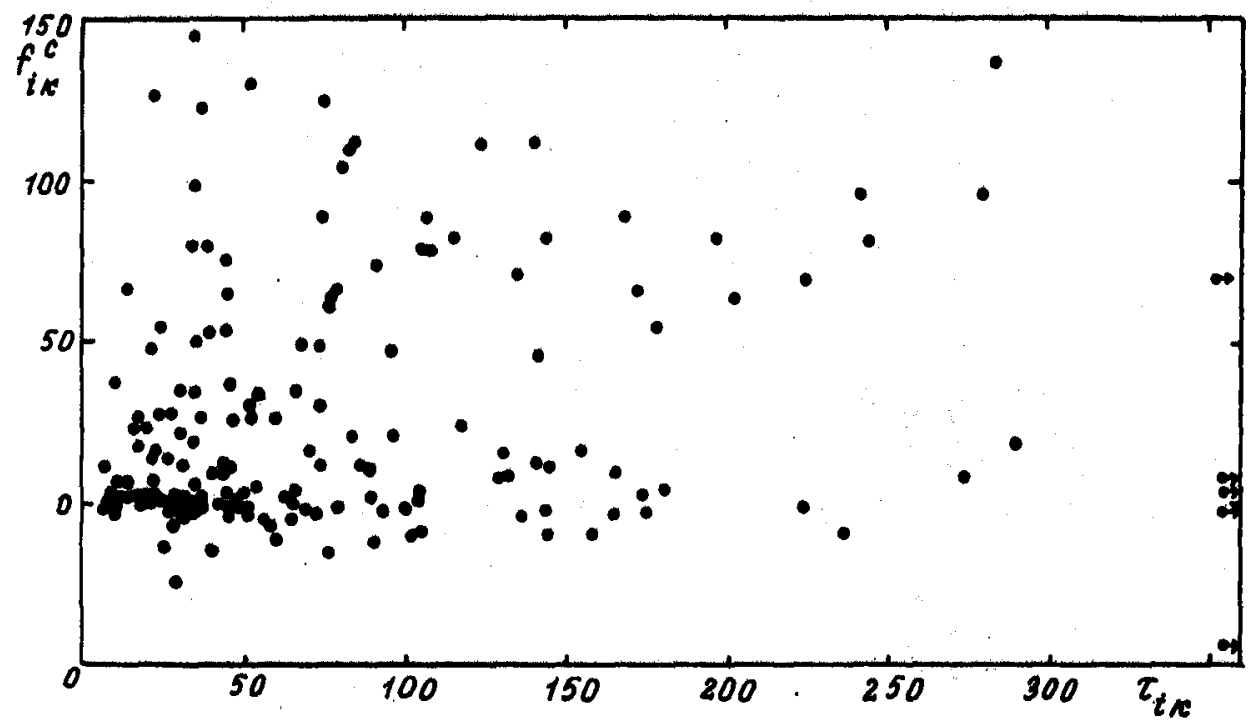

Fig. 6. Relation between orbital mass-to-luminosity ratio and linear projected separation for galaxies in the triplets. Arrows show the pairs with separation greater than 350 kpc.

\section{CROSSING TIME}

When calculating virial masses it is necessary to make certain, that triplets of galaxies had enough time for dynamical relaxation. Using the average projected harmonic radius of a triplet $r_{H}$ and rms velocity along the line of sight $S_{v}$, we determined from eq. (8) the dimensionless crossing time of the system $\tau$. The distribution of triple galaxies in $\log \tau$ is shown in the upper part of Figure 7. It has quite a symmetric shape with median $\tau_{0.5}=$ $\operatorname{dex}-1.40$.

Another estimate of $\tau$ was obtained by considering pairwise combinations of galaxies in the triplet. If the motion of two galaxies is circular, the rotation period around their common gravitation center can be expressed as

$$
\tau_{i k}=4 \mathrm{H} r_{i k} /\left(\mathrm{V}_{\mathrm{i}}-\mathrm{V}_{\mathrm{k}}\right)
$$

where the numerical value of the coefficient was obtained by assuming chaotic orientation of orbits relative to the line of sight. The distribution of dimensionless rotation periods is shown on a logarithmic scale in the lower histogram of Figure 7. Some of the pairs formed from triplet members have rotation periods exceeding the Hubble time. In this region $(\tau>1)$ the cases prevail for which when projected velocity difference for two galaxies is less than its measurement error (hatched). The median orbital period is dex -1.05 . 


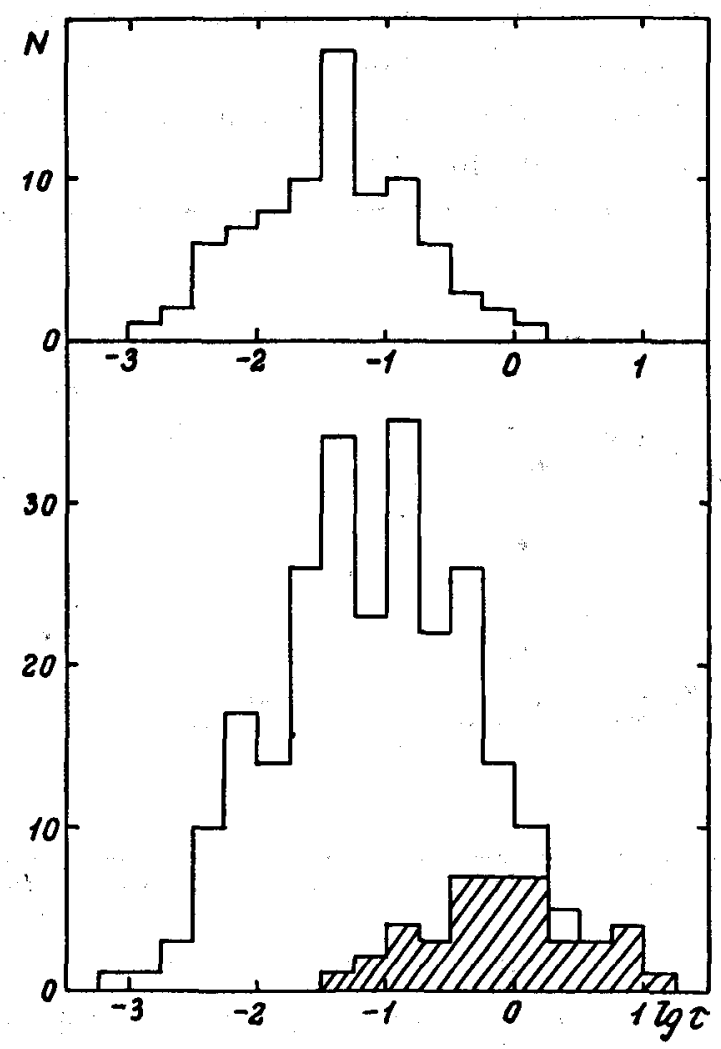

Fig. 7. Triple-system distribution according to crossing time (top) and orbital period (below) in units of the Hubble time, $\mathrm{H}^{-1}$. Hatching shows the pairs in which the radial velocity difference is less than its measurement error.

It should be noted that crossing time might be determined by using the observed values in different ways. Thus, Gott et al. (1973) used a "virial" crossing time, which is less than the one calculated according to eq. $(8)$ by $(20 \sqrt{ } 5 / 3 \pi) \approx 4.7$ times. Jackson (1975) suggested expressing the estimate of $\tau$ through the RMS system radius and $S_{v}$ ("inertial" crossing time). Gott and Turner (1974) prefer a "linear" time $\tau_{\mathrm{L}}=(2 \mathrm{H} / \pi)<$ $r_{i k} /\left(<V_{i}-V_{k}>\right)$, which is less sensitive to the presence of closely projected pairs and background objects. According to numerical simulation of the collapse and relaxation of a group of galaxies (Gott and Turner, 1977), a triple system reaches virial equilibrium when its crossing time $\tau=2 \mathrm{Hr}_{H} / \mathrm{S}_{\mathrm{v}} \lesssim 0.4$. As is seen from Figure 7, about $95 \%$ of triplets satisfy this condition; in other words, almost all the triple systems have already passed the initial period of their dynamical evolution.

\section{CONCLUSION}

Using various ways to estimate virial mass and various methods of excluding optical systems we found that the median mass-to-luminosity ratio for the sample of 84 triplets ranges from 20 to $30 f_{\odot}$. This value is $3-4$ times less than the one obtained for triple systems from the lists of groups by Geller, Huchra (1983) and Vennik (1986). But it is 4 times as large as the median mass-to-luminosity ratio $\left(\approx 6 f_{\odot}\right)$ which follows from rotation 
curves of separate galaxies or analysis of orbital motions in pairs.

To explain this excess it is sufficient to suppose that about $80 \%$ of triplets' total mass is hidden matter. But there are serious grounds for believing that the sample considered is strongly contaminated by false non-isolated "triplets", which are fragments of systems with higher multiplicity. Therefore all this virial excess may be an "artifact", originating from the extreme rareness of true autonomous triple systems.

\section{REFERENCES}

Anosova J.P., Orlov V.V. 1985, Uchenye Zapiski LGU (Leningrad State University), mathematics, 82, No 416, 66-144.

Bahcall J.N., Tremaine S. 1981, Astrophys. J. 224, 805-820.

Faber S.M., Gallagher J.S. 1979, Ann. Rev. Astron. Astrophys. 17, 135-187.

Gott J.R., Turner E.L. 1977, 213, 309-322.

Gott J.R., Wrixon G.T., Wannier P. 1973, Astrophys J. 186, 777-785.

Jackson J.C. 1975, Mon. Not. R. Astron. Soc. 173, 41.

Karachentsev I.D. 1972, Catalog of isolated pairs of galaxies in the northern hemisphere, Soobshch. Spets. Astrofiz. Obs. Akad. Nauk USSR, 7, 1-91.

Karachentsev I.D., Shcherbanovskij A.L. 1978, Soobshch. Spets. Astrofiz. Obs. 24, 5-46. Karachentsev I.D. 1985, Astron. Zh. 62, 417-431.

Karachentsev I.D. 1987, Binary galaxies (Dvoinye Galaktiki), Moscow, Nauka.

Karachentsev I.D., Karachentseva V.E., Lebedev V.S. 1989, Izv. Spets. Astrofiz. Obs. Akad. Nauk USSR, 27, 67.

Karachentseva V.E., Karachentsev I.D., Shcherbanovskij A.L. 1979, Izv. Spets. Astrofiz. Obs. Akad. Nauk USSR, 11, 51.

Karachentseva V.E., Karachentsev I.D. 1982, Astrofizika, 18, 5-16.

Karachentseva V.E., Karachentsev I.D., Lebedev V.S. 1988, Izv. Spets. Astrofiz. Obs.

Akad. Nauk USSR, 26, 42.

Limber D.N., Mathews W.G. 1960, Astrophys. J. 132, 286.

Trimble V. 1987, Ann. Rev. Astron. Astrophys. 25, 425-472.

Vaucouleurs G. de, Vaucouleurs A. de, Corwin H.C. 1976, Second Reference Catalogue of Bright Galaxies, Univ. of, Texas Press, Austin.

Vennik, J.A. 1986, Astron. Nachr. 307, 157.

TABLE 1

MEDIAN VIRIAL MASS-TO-LUMINOSITY RATIO FOR 84 TRIPLETS

\begin{tabular}{ccccccc} 
Estimator & \multicolumn{2}{c}{ Whole Sample } & \multicolumn{2}{c}{ Objects with $\mathrm{f}<10^{3}$} & \multicolumn{2}{c}{ Objects with $\Delta \mathrm{V}_{i k}<500 \mathrm{~km} / \mathrm{s}$} \\
& $\log f_{0.5}$ & $\log f^{\mathrm{c}}{ }_{0.5}$ & $\log f_{0.5}$ & $\log f^{c}{ }_{0.5}$ & $\log f_{0.5}$ & $\log f_{0.5}^{c}{ }_{0.5}$ \\
A & 1.83 & 1.76 & 1.53 & 1.46 & 1.49 & 1.43 \\
W & 1.70 & 1.66 & 1.49 & 1.46 & 1.49 & 1.43 \\
P & 1.73 & 1.69 & 1.44 & 1.38 & 1.35 & 1.30 \\
Pairs $(n=585)$ & 0.93 & 0.86 & 0.83 & 0.70 & 0.75 & 0.60
\end{tabular}


Plate 1. Two examples of triple systems having a moderate virial mass-to-luminosity ratio $\left.\left(\approx 100 \mathrm{f}_{\odot}\right) . a\right)$ T $50=$ Hickson 68 with radial velocities $+2410,+2115$, and $+3096 \mathrm{~km} / \mathrm{s}$ for $\mathrm{A}, \mathrm{B}, \mathrm{C}$-components, respectively.

b) T $83=$ Hickson 99 with radial velocities $+9084(\mathrm{~A}),+9066(\mathrm{~B})$, and $+8259(\mathrm{C}) \mathrm{km} / \mathrm{s}$. Both photographs in blue light were obtained by N. Tikhonov, with the 6-meter telescope.

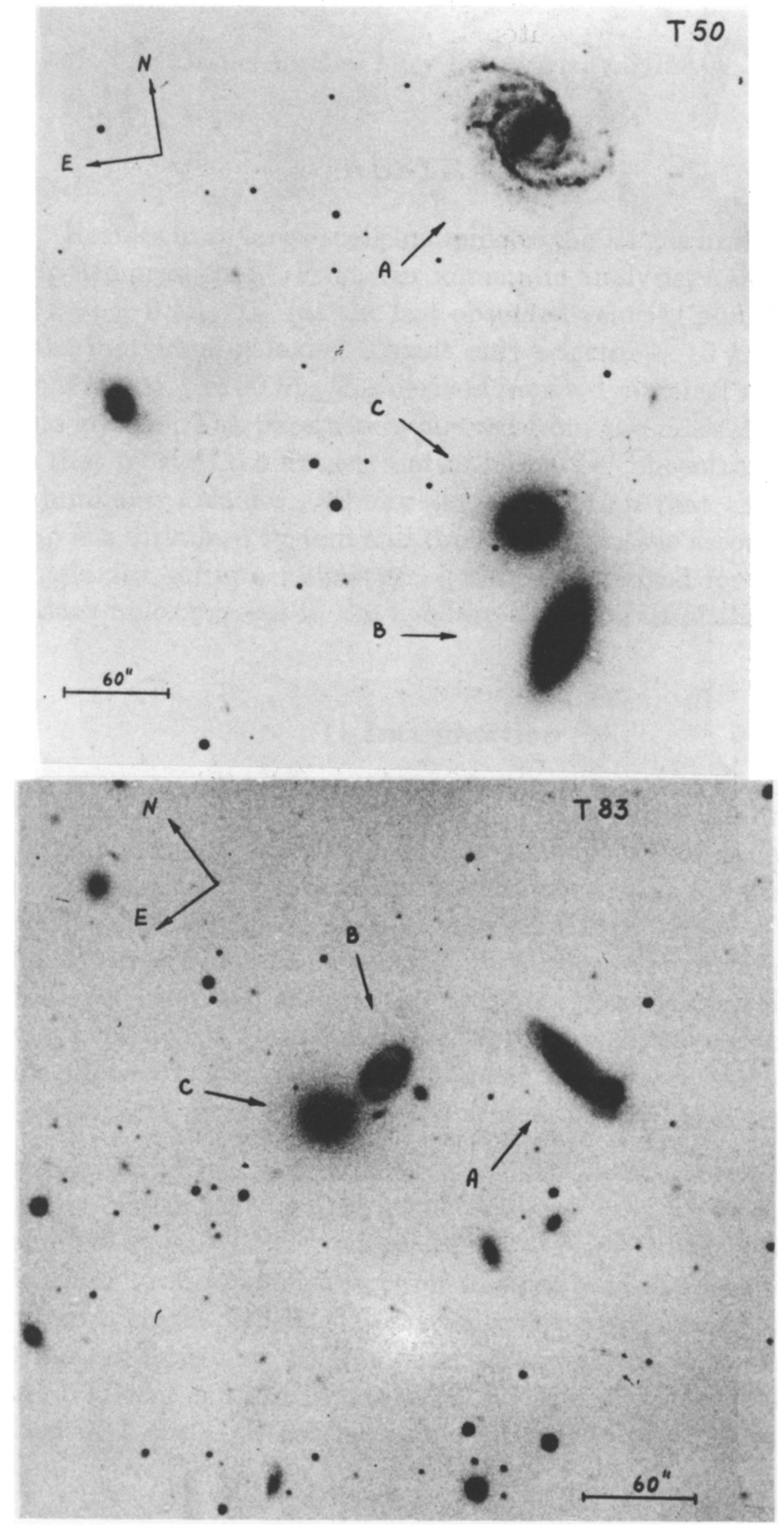

